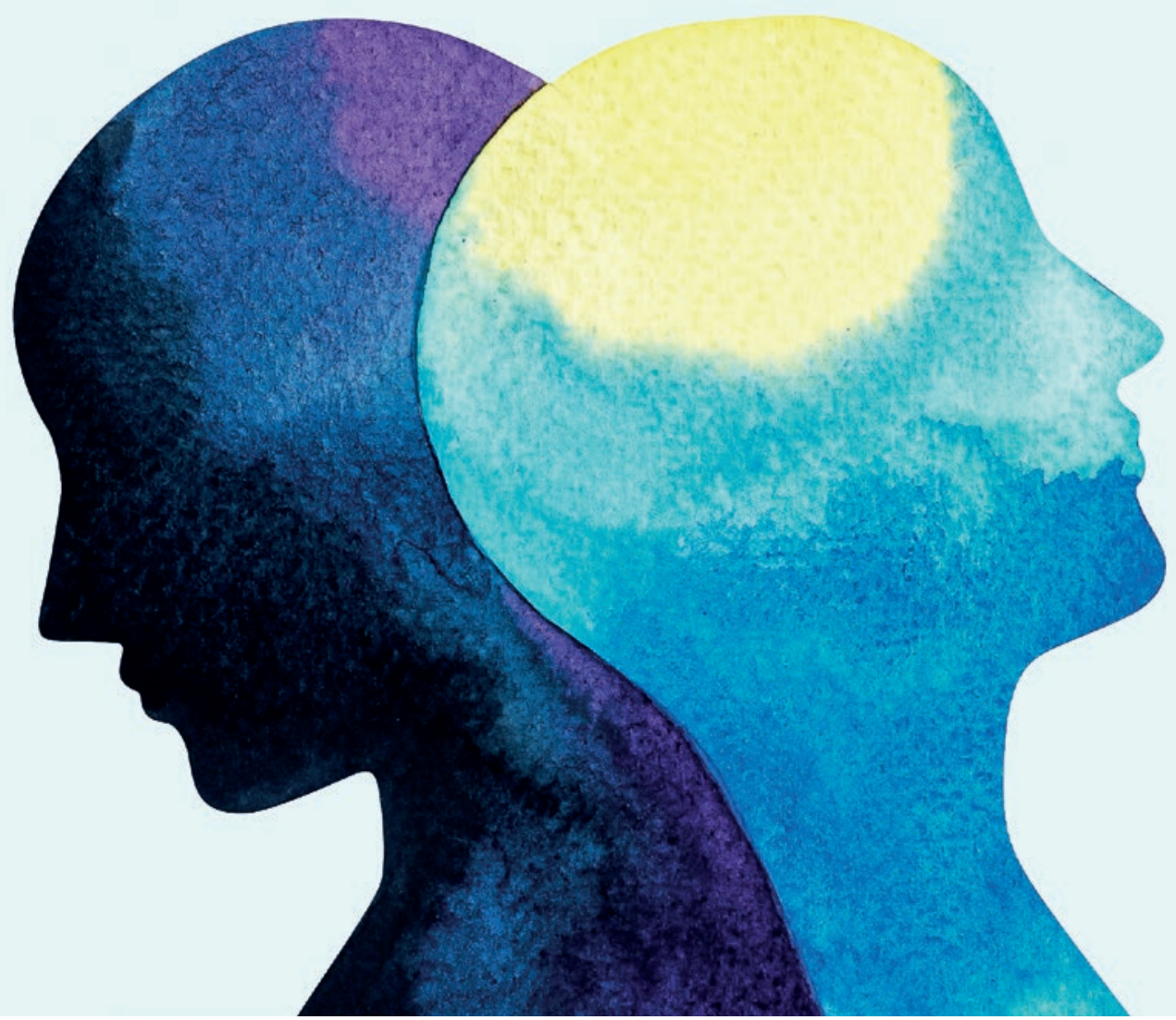

La structure tarifaire utilisée pour la prise en charge des personnes atteintes de maladies psychiques a été remaniée.

\title{
TARPSY 4.0 poursuit sur une voie qui a fait ses preuves
}

\section{Bruno Trezzini}

Dr phil., expert, division Médecine et tarifs hospitaliers, FMH

La quatrième version de TARPSY a été remise au Conseil fédéral pour approbation. Si le lien entre tarif et prestations a été amélioré, la prise en compte du degré de sévérité des cas peut devenir encore plus précise. Au vu de la situation, les données de l'année 2020 seront peu sûres, ce qui a incité SwissDRG SA à suspendre pendant un an le développement de TARPSY. Par conséquent, TARPSY 4.0 restera en vigueur jusqu'en 2023.

TARPSY 4.0 a été développé par SwissDRG SA sur la base des données de coûts et de prestations de l'année 2019 [1]. Si cette version est approuvée par le Conseil fédéral, elle entrera en vigueur le $1^{\text {er }}$ janvier 2022. Le conseil d'administration de SwissDRG SA a par ailleurs décidé le 11 juin 2021 de renoncer au développement de TARPSY sur la base des données de 2020 [2]; notamment parce qu'elles risquent d'être biaisées en raison de la pandémie et que des effets négatifs sur la facturation des cas de psychiatrie hospitalière ne peuvent pas 
être totalement exclus. De ce fait, la version 5.0 de TARPSY sera calculée sur la base des données de 2021 et préparée pour une entrée en vigueur en 2024; TARPSY 4.0 sera donc encore utilisé en 2023. Dans ces colonnes, nous présentons quelques points capitaux de TARPSY en général et de TARPSY 4.0 en particulier.

\section{La qualité des données: une nécessité pour une rémunération appropriée}

Quand on regarde les données collectées de 2016 à 2019, on remarque que la part de cas plausibles pouvant être utilisés pour le développement du tarif s'est établie à $75 \%$ chaque année et que les cas exclus le sont principalement lorsque les coûts de traitements et de soins infirmiers sont inhabituellement faibles ou que la saisie des médicaments et des formulaires HoNOS/ CA est incomplète voire incorrecte [3]. Le fait qu'un

Les modèles de documentation CHOP de la FMH facilitent la saisie des prestations médicales et des plans de traitements multimodaux.

quart des données doivent être supprimées après plusieurs années de travail avec TARPSY rappelle qu'il est nécessaire de veiller à une meilleure saisie. En effet, disposer de données de qualité permet de mieux recenser les prestations fournies et pose ainsi les bases d'une rémunération appropriée. Il est primordial que les cliniques profitent davantage de l'offre de SwissDRG SA qui propose de contrôler leurs données en cours d'année [4]. Les modèles de documentation CHOP mis à disposition par la FMH facilitent quant à eux la saisie des prestations médicales complexes et des plans de traitements multimodaux [5]. La FMH est par ailleurs à la disposition de ses membres pour répondre à leurs questions sur les codes de la CHOP ou sur les directives de codage.

\section{Publication souhaitable des résultats du codage}

Jusqu'à présent, peu d'informations relatives à la fréquence des codages [6] spécifiques à la psychiatrie (CHOP) et à leur qualité sont disponibles. Quelques institutions publient un condensé de leurs résultats sur leur site internet [7]. Il serait souhaitable que davantage de cliniques suivent ces exemples réjouissants. Par ailleurs, l'accès public à une évaluation systématique de tous les rapports de révision du codage d'une même année permettrait d'obtenir une meilleure vue d'ensemble des progrès qualitatifs du codage.

\section{Quatre codes CHOP psychiatriques supplémentaires ont été déterminants pour le tarif}

Les codes CHOP spécifiques à la psychiatrie ont eu leur premier impact tarifaire dans la version TARPSY 3.0 dans laquelle ils ont servi de critères de séparation des coûts ou permis d'établir des rémunérations supplémentaires [8]. Cette évolution s'est poursuivie avec les quatre codes psychiatriques supplémentaires de la version TARPSY 4.0, dans laquelle ils ont été déterminants en tant que critères de séparation des coûts [9].

La forme sévère d'une maladie combinée à une éventuelle polymorbidité exige généralement un nombre de prestations supérieures à la moyenne. La rémunération de ces cas devrait donc être plus élevée que celle des cas moins complexes. Elle peut se présenter, d'une part, sous la forme de rémunérations supplémentaires [10]. D'autre part, TARPSY permet d'établir un lien avec le degré de sévérité d'un cas grâce notamment à la saisie de la gravité des symptômes (HoNOS/CA [11]) mais aussi des limitations fonctionnelles, qu'elles soient motrices ou cognitives (codes de diagnostics U5O et U51).

\section{Un tarif plus en lien avec les prestations et le degré de sévérité}

Les sociétés de discipline concernées expriment cependant des réserves quant à l'utilisation des codes de diagnostics U50 et U51 dans leur forme actuelle. Par contre, le fait de pouvoir à l'avenir utiliser le MoCA [12], en plus de l'indice de Barthel étendu, du FIM cognitif [13] et du MMSE [14], pour la saisie des limitations fonctionnelles cognitives correspond davantage à leurs attentes. Cela sera en effet possible grâce au transfert des valeurs du MoCA vers celles du MMSE [15]. En revanche, il manque toujours une échelle permettant de déterminer la sévérité d'un cas en tenant compte des multiples diagnostics secondaires. Heureusement, SwissDRG SA prévoit, entre autres, d'utiliser les ressources libérées par le report de la version TARPSY 5.0 pour faire avancer une nouvelle logique concernant le niveau de sévérité dans le domaine de la psychiatrie hospitalière.

\section{Solution en cours pour les prestations intercurrentes}

Concernant la rémunération des prestations intercurrentes, ce qui est déclaré à ce sujet dans la «Convention de structure tarifaire TARPSY» [16] diffère des «Règles et définitions pour la facturation des cas selon SwissDRG et TARPSY». Initialement, les partenaires tari- 
faires avaient prévu de mettre sur pied d'ici la fin juin 2021 une convention nationale réglementant aussi bien pour TARPSY que pour ST Reha la facturation supplémentaire mais séparée des prestations médicales découlant d'une affection apparue en cours de traitement hospitalier [17]. Si les travaux ont duré plus longtemps que prévu, il semblerait que les partenaires tarifaires seraient sur le point de la finaliser.

\section{Conclusion: le système continue d'évoluer}

TARPSY 4.0 poursuit son évolution sur une voie qui a déjà fait ses preuves. Dans ce développement, les différentes procédures de demandes [18] revêtent une importance capitale afin de viser une rémunération toujours

\section{Les procédures de demandes revêtent une} importance capitale afin de viser une rémunération adaptée aux prestations fournies.

plus adaptée aux prestations fournies. Conjointement avec les sociétés de discipline, la FMH œuvre activement à l'élaboration de codes CHOP spécifiques à la psychiatrie (procédure de demande de l'OFS) et dépose des suggestions en vue d'adapter la structure tarifaire TARPSY (procédure de demande de SwissDRG SA). La FMH apporte un soutien actif aux membres qui souhaitent déposer une demande. En outre, les ateliers proposés par SwissDRG SA aux fournisseurs de prestations offrent la possibilité de promouvoir une meilleure compréhension entre les différents partenaires et de maintenir l'aspect évolutif et adaptatif du système [19].

FMH

Division Médecine et tarifs

hospitaliers

Baslerstrasse 47

CH-4600 Olten

Tél. 0313591111

Fax 0313591112

tarife.spital[at]fmh.ch

\section{Crédits photo}

Benjavisa Ruangvaree | Dreamstime.com

\section{Bibliographie et remarques}

1 www.swissdrg.ch $\rightarrow$ Psychiatrie $\rightarrow$ Système TARPSY 4.0/2022

2 www.swissdrg.ch $\rightarrow$ Portrait $\rightarrow$ Conseil d'administration $\rightarrow$ Communication
3 SwissDRG SA. TARPSY 4.0: rapport sur le développement de la structure tarifaire. 2021: p. 3 (www.swissdrg.ch $\rightarrow$ Psychiatrie $\rightarrow$ Système TARPSY 4.0/2022 $\rightarrow$ Documents complémentaires).

4 www.swissdrg.org $\rightarrow$ Psychiatrie $\rightarrow$ Relevé des données $\rightarrow$ Relevé 2022 (données 2021).

5 www.fmh.ch $\rightarrow$ Prestations $\rightarrow$ Tarifs hospitaliers $\rightarrow$ Modèles de documentation CHOP.

6 SwissDRG SA publie un explorateur de données qui met l'accent sur les groupes de coûts psychiatriques (PCG) et ne laissant que des possibilités limitées de filtre selon les codes de la CHOP (www. swissdrg.ch $\rightarrow$ Psychiatrie $\rightarrow$ Système TARPSY 3.0/2021 $\rightarrow$ Explorateur de données).

7 Cf. www.upk.ch $\rightarrow$ Über uns $\rightarrow$ Qualität $\rightarrow$ TARPSY. Le «Règlement concernant l'exécution de la révision du codage selon TARPSY» (www.swissdrg.ch $\rightarrow$ Psychiatrie $\rightarrow$ Système TARPSY 4.0/2022 $\rightarrow$ Révision du codage) prévoit de rendre accessible sous forme de résumé les évaluations effectuées par les cliniques.

8 Trezzini B, Meyer B. TARPSY 3.0, avec un lien plus étroit aux prestations. BullMed. 2020;101(19-20):607-609.

9 Il s'agit de: $94.3 \mathrm{~B}$ «Traitement complexe du patient psychiatrique gériatrique polymorbide en soins aigus»; $94.3 \mathrm{C}$ «Traitement complexe lors de démence avec complications psychiatriques et psycho-organiques»; 94.3G «Traitement complexe lors d'anorexie en psychiatrie»; 94.4A «Traitement mère-enfant en psychiatrie adulte». Cf. SwissDRG SA. "Présentation du système TARPSY 4.0" 2021 (www.swissdrg.ch $\rightarrow$ Psychiatrie $\rightarrow$ Système TARPSY 4.0/2022 $\rightarrow$ Documents complémentaires).

10 TARPSY applique des rémunérations supplémentaires à la psychiatrie en plus de celles utilisées en soins somatiques aigus (www. swissdrg.ch $\rightarrow$ Psychiatrie $\rightarrow$ Système TARPSY 4.0/2022 $\rightarrow$ Catalogue PCG).

11 Health of the Nation Outcomes Scales/ Health of the Nation Outcomes Scales for Children and Adolescents.

12 Montreal Cognitive Assessment.

13 Functional Independence Measure.

14 Minimal Mental State Examination.

15 OFS. Circulaire pour les codeuses et codeurs 2021, n². 2021: p. 13 (www.bfs.admin.ch $\rightarrow$ Trouver des statistiques $\rightarrow$ Catalogues et banques de données $\rightarrow$ Nomenclatures $\rightarrow$ Classifications et codage médicaux).

16 www.swissdrg.ch $\rightarrow$ Psychiatrie $\rightarrow$ TARPSY $\rightarrow$ Convention de struc ture tarifaire.

17 SwissDRG SA. Règles et définitions pour la facturation des cas selon SwissDRG et TARPSY. 2021: p. 16 (www.swissdrg.ch $\rightarrow$ Psychiatrie $\rightarrow$ Système TARPSY 4.0/2022 $\rightarrow$ Règles et définitions).

18 Rufer M. Évolution active du tarif par le dépôt de propositions. BullMed. 2021;102(21):684-686

19 P. ex. lors de l'atelier TARPSY sur le thème "Mécanismes de développement, approbation et mise en œuvre» (www.swissdrg.org $\rightarrow$ Portrait $\rightarrow$ Manifestations $\rightarrow$ TARPSY Workshop 2021). 